\title{
MULTIPLE CHOICE QUESTION ON EPILEPSY SURGERY (CORRECT ANSWER IS IN BOLD AND UNDERLINED)
}

Q.1 If the patient has failed 2 drugs in their appropriate combination and dosage, the likely chance of the third drug to control epilepsy would be
A) $\leq 5 \%$
B) $10-20 \%$
C) $20-30 \%$
D) $40-50 \%$

Q.2 Which of the following facts about drug resistant epilepsy (DRE) in NOT true?
A) DRE occurs as a result of long standing acquired drug resistance
B) DRE is do novo and can be identified early in the course of disease
C) DRE may be suspected when the response to first drug fails
D) The only viable option for DRE is epilepsy surgery

Q.3 Which of the following is NOT true about epilepsy surgery?
A) This is a surgery performed primarily for curing/controlling drug resistant epilepsy
B) MRI has to show a lesion for the surgery to be successful
C) Multi-modal imaging clearly identifies the epileptogenic networks
D) The 10 year seizure free outcome for mesial temporal sclerosis ranges from $50-60 \%$

Q.4 Which of the following is NOT a curative surgery?
A) Hemispherotomy
B) Multiple sub-pial transection
C) TPO disconnection
D) Lesionectomy

Q.5 Which of the following is NOT true about hippocampal sclerosis surgery?
A) Recent meta-analysis has shown better outcome for a standard temporal lobectomy combined with amygdalo-hippocampectomy when compared to selective amygdalo-hippocampectomy
B) Hippocampus has to be resected upto the level of superior colliculus to ensure better outcomes
C) Both dorsal and ventral amygdala has to be excised to ensure a seizure free outcome
D) The adjacent structures like entero-rhinal cortex, uncus, para-hippocampal cortex also has to be excised

Q.6 Which of the facts in NOT true about hemispherotomy?
A) Functional hemispherotomy was first described by Rasmussen
B) There are basically 2 techniques for hemispherotomy- per-insular and vertical trans-cortical
C) Hemispherotomy provides one the best outcomes in epilepsy surgery
D) The chief reason that the anatomical hemispherectomy was given up was due to post operative hydrocephalus


Q.7 Which of the following is the most relevant indication for corpus callosotomy?
A) Lenox-Gestaut with drop attacks
B) DRE without any localization
C) Infantile spasms
D) Myoclonic jerks

Q.8 Which of the following is NOT true about surgical aspect of focal cortical dysplasia?
A) Electro corticography is useful for localization and resection
B) Trans mantle sign is found in Type I Palmini
C) FCD may be associated with certain tumors
D) Hemi-megelencephaly is a very severe form of FCD

Q.9 Which of the following is NOT true about vagal nerve stimulation?
A) VNS should be treated like another drug
B) VNS leads to about $50 \%$ seizure free outcome in about $50 \%$ of cases
C) VNS has similar indications as for copuscallosotomy
D) VNS may be also indicated in certain lesional cases

Q.10 Which of the following is NOT true about SEEG?
A) It may be performed using both a robotic device and standard stereotactic frame even the former is more convenient
B) Has lesser morbidity than standard depth electrodes
C) Is performed using a burrhole
D) Since it is fitted using an anchor bolt, may be explanted easily 